\title{
Current standards of neuropsychological assessment in epilepsy surgery centers across Europe
}

Author information:

Viola Lara Vogt ${ }^{\mathrm{a}}$ Petia Dimova ${ }^{\mathrm{b}}$, Hrvoje Hecimovic ${ }^{c}$, Einar Heminghyt ${ }^{\mathrm{d}}$, Alena Javurkova ${ }^{\mathrm{e}}$, Mary H. Kosmidis ${ }^{\dagger}$, Sofia Ljunggren ${ }^{g}$, Patricia Martin-Sanfilippo ${ }^{h}$, Federica Pellei, Philippe Ryvlin', Elisabeth Schmidk, Monique van Schooneveld', Anne-Sophie Wendling ${ }^{\mathrm{m}}$, JuriAlexander Witt ${ }^{\mathrm{a}}$, Christoph Helmstaedter ${ }^{\mathrm{a}}$

a Department of Epileptology, University of Bonn, Medical Center, Sigmund Freud Straße 25, 53105 Bonn, Germany.

${ }^{b}$ Clinic of Child Neurology, St Naum University Hospital of Neurology and Psychiatry, 1 Lyuben Rusev street, Sofia, Bulgaria

c Zagreb Epilepsy Center, Department of Neurology, University Hospital, Vinogradska cesta 29, 10000, Zagreb, Croatia

d National Centre for Epilepsy (SSE), Oslo University Hospital, Norway

${ }^{e}$ Charles University in Prague, 2nd Faculty of Medicine, Motol University Hospital, V Úvalu 84, Prague 5, CZ 150 06, Czech Republic

${ }^{\mathrm{f}}$ Aristotle University of Thessaloniki, Thessaloniki 54124, Greece.

${ }^{9}$ Department of Clinical Neuroscience and Rehabilitation, Institute of Neuroscience and Physiology, The Sahlgrenska Academy at the University of Gothenburg, Blå Stråket 7, Sahlgrenska University Hospital, 41345 Gothenburg, Sweden

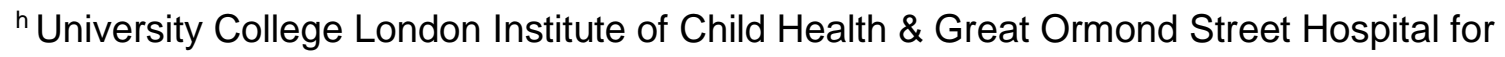
Children NHS Foundation Trust, London, United Kingdom

i Claudio Munari Epilepsy Surgery Centre, Niguarda Hospital, Piazza Ospedale Maggiore 3, 20162, Milan, Italy

'Institute for Epilepsies (IDEE), Lyon's Neuroscience Center, INSERM U1028

Lyon, FR

k Department of Neurology, Paracelsus Medical University Salzburg, Christian Doppler Klinik, Salzburg, Austria

'Department of Child Neurology, Brain Center Rudolf Magnus, University Medical Center Utrecht, The Netherlands

m Epilepsiezentrum Kork, Landstraße 1, 77694 Kehl-Kork, Germany 


\section{MANUSCRIPT DATA:}

Title: Current standards of neuropsychological assessment in epilepsy surgery centers across Europe

Running title: European standards of neuropsychological assessment in epilepsy

Title: 82 characters

Abstract: 247 words

Main body text: 5255 words

No. of references: 34

Tables: 7

Figures: 0

\section{Corresponding author:}

Viola Lara Vogt

Department of Epileptology

University of Bonn

Sigmund Freud Straße 25

53105 Bonn, Germany.

Tel: $0042-0228-287-14436$

Fax: $0042-0228-287-14486$

E-Mail: viola.vogt@ukb.uni-bonn.de 


\section{ABSTRACT}

Background: Aiming at the harmonization and establishment of common standards in the neuropsychological assessment of surgical patients with epilepsy, the current practice in European epilepsy centers was evaluated.

Method: Twenty-six epilepsy centers joining "E-PILEPSY", an EU funded pilot network of European reference centers for drug resistant epilepsy and epilepsy surgery, were asked to report the status of neuropsychological assessment in adults and children via two different surveys.

Results: It was agreed that neuropsychology is an essential part of presurgical work-up. Large agreement was found on indications (localization, epileptic dysfunctions, adverse drugs effects, postoperative monitoring) and the domains to be evaluated (memory, attention, executive functions, language, visuo-spatial skills, intelligence, depression, anxiety, and quality of life). Although 186 different tests are in use, a core group of tests reflecting minimal agreement could be discerned. Variability exists with regard to indications, protocols and paradigms for the assessment of hemispheric language dominance. For the tests in use, little published evidence of clinical validity in epilepsy was provided. Participants of the survey reported a need for improvements concerning the validity of the tests, tools for the assessment of everyday functioning and accelerated forgetting, national norms and test conormalization.

Conclusion: Based on this survey, a consensus on the indications and principles of neuropsychological testing was achieved. Despite the variety of tests in use, the survey indicated there may be a core set of tests which appear to result from experience as well as published evidence. By combining with the results of an ongoing systematic literature review, we aim for a battery which can be recommended for the use across epilepsy surgical centers in Europe.

Keywords: Epilepsy surgery; Neuropsychology; Diagnostic, Consensus, Europe 


\section{Introduction}

In January 2014, a European pilot network of reference centers in drug resistant epilepsy and epilepsy surgery, funded by the European Union in the framework of the Health Program (http://www.e-pilepsy.eu), was established. The primary objective is to improve the quality, accessibility and harmonization of presurgical diagnostic approaches in patients with drug resistant focal epilepsy across Europe. One aim of this network is to describe the requirements and the structure of epilepsy surgical centers and of current practice in brain imaging, source localization procedures [1], EEG monitoring [2] and, what will be dealt with here, the neuropsychological assessment and outcome monitoring in patients with drug resistant epilepsy.

Epilepsy surgery is an elective procedure with the ultimate goal of improving the individual health-related quality of life through sustained seizure-freedom or improvement. At the same time, successful epilepsy surgery aims at preservation or even improvement of the patients' functional capabilities, mood and behavior, thereby improving their socio-economic opportunities and quality of life. In the context of epilepsy surgery, neuropsychology aims to identify epilepsy-related cognitive impairments and their etiological attribution to lesions, the active epilepsy, drug treatment, and mood within a neuropsychological developmental framework which takes age at onset and the brain's capacity for reorganization and compensation into consideration [3].

Epilepsy surgery can be associated with cognitive decline. Before surgery, up to $80 \%$ of patients already show cognitive impairment in at least one neuropsychological domain [4]. After temporal lobe resections, the most frequent type of surgery, up to $45 \%$ may experience a decline in memory $[5,6]$. Furthermore, in terms of complications, surgery can lead to new cognitive deficits or an unexpectedly severe deterioration of cognition, for example disabling 
memory deficits after temporal lobe surgery [7]. In children, surgery has an impact on postsurgical cognitive development, which is often positive [8]. Surgically caused impairments are especially intolerable in the absence of postsurgical seizure freedom $[9,10]$. As such, it is important to individually predict postsurgical deficits to a certain degree. Major predictors of neuropsychological outcome include the presurgical performance, reflecting functional integrity of the resected tissues on the one hand, and reserve capacities of nonaffected brain structures on the other hand. Additional predictors are cerebral functional plasticity, seizure control and the burden of antiepileptic drug treatment [6, 11-14]. Neuropsychological examinations after surgery help to objectively quantify cognitive outcomes, indicate the need for rehabilitative care, and, in terms of quality control, help to optimize treatment outcome of resective epilepsy surgery with minimal cognitive risks $[6,15]$.

Apart from cognitive problems, affective problems such as depression and anxiety in adults as well as autism and attention deficit hyperactivity disorder (ADHD) in children are very frequent [16]. Affective problems are seen in up to $50 \%$ of surgical candidates and - similar to cognitive and behavioral problems - in part result from epilepsy and its treatment, and in part may share the same underlying pathophysiology with the epilepsy [17]. They are often under-diagnosed and need to be considered and treated [18]. On a superordinate level quality of life has been identified as a relevant outcome parameter [19].

The aforementioned aspects are all central to be assessed and evaluated in the context of epilepsy surgery. This survey describes how this is being achieved in different reference centers for epilepsy surgery across Europe. While the neuropsychology task force of the International League against Epilepsy (ILAE) has recently updated the concepts and principles of neuropsychological assessments in epilepsy [20], detailed information about which tests to use is less easy to obtain. Earlier surveys 23 years [21], 11 years [22] and five years ago $[23,24]$ indicate that there is a wide range of neuropsychological tests used in different epilepsy clinics, not to mention in different countries [25]. In a review of the 
theoretical and practical issues of neuropsychology in epilepsy we postulated an exemplary evidence-based, question guided and modular approach which is not only descriptive but can make a difference for patients and their treatment [26]. For the purpose of E-PILEPSY, however, such a model needs to be filled with content which is acceptable not only on a national basis $[27,28]$ but across different centers in different countries.

Communication of the neuropsychological features of patient groups, of surgical methods and their outcomes across centers and countries calls for a common language. This requires a harmonization of procedures. These procedures need to meet certain methodological criteria such as age-corrected normative data, standardization for repeated measurement, and sensitivity to the factors causing cognitive impairments in epilepsy.

One central and explicit aim of the E-PILEPSY project is to harmonize best practice in epilepsy surgery and develop guidelines and recommendations based on best available evidence. Given historical and legal reasons but also cultural and linguistic diversities, it is an ambitious undertaking to strive for a European standard. As a first step, the current practice in the E-PILEPSY associated reference centers needs to be documented. In order to obtain that information we conducted a survey among all centers participating in the E-PILEPSY project. The second step, which has already been initiated and which will be dealt with in subsequent publications, is a systematic review of the existing literature in order to discern which instruments may best serve the objectives of neuropsychology in the context of epilepsy surgery. In a third step a selection of tests will be made which then requires efforts of normalization and standardization for repeated measurement in each associated European country. In parallel, work on a common web-based database, which will provide tests, materials and normative data, is in progress.

\section{Methods}


Two separate surveys were sent out electronically to 26 epilepsy surgery centers of the EPILEPSY network (21 countries, 19 languages: Austria Salzburg, Belgium Gent, Bulgaria Sofia, Croatia Zagreb, Czech Republic, Prague, Finland Kuopio, France ?, Germany Bonn and Kork, Greece Thessaloniki, Hungary Budapest, Italy Milano, Lithuania Vilinus, Netherlands Utrecht, Norway Oslo, Romania Bucharest, Russia Moscow, Spain Madrid, Sweden Goteborg, Switzerland Genf, Turkey Istanbul, UK London). The first survey focused on neuropsychological testing per se and was - in part - similar to the German inquiry in 2008[29]. The focus was in interictal, pre- and postsurgical assessment of patients with epilepsy, not on bed-side, ictal testing. The second inquiry was related to the assessment of hemispheric dominance for language functions. The surveys were launched in February 2014 and concluded in June 2015. The first survey consisted of 29 questions addressing the neuropsychological assessment per se. The questions comprised multiple choice answers and/or free text fields and covered the following areas: Availability of epilepsy surgery and of neuropsychological service for adults and children, implementation, organization and capacity of the neuropsychological service, indications for the neuropsychological assessment, consequences for clinical decision making and counseling, psychiatric assessment, as well as detailed descriptions of tests and questionnaires that are in use including published evidence for their use in patients with epilepsy.

The second survey on the assessment of hemispheric dominance for language functions was sent out in August 2014 and concluded in June 2015. This questionnaire comprised 12 items centering on the most common methods (IAT, intracarotid amobarbital test and fMRI, functional magnetic resonance imaging) and included one question on the general decision process of when and how to assess language dominance.

The surveys were completed by neuropsychologists $(n=14)$ or neurologists $(n=11)$, with information provided by the neuropsychologists since they were no official participants in the project. 
We excluded our own center (Bonn) concerning one item of the survey asking for references of studies proving the validity of the neuropsychological tools for the use in epilepsy. The reason for this decision was that as coordinator of the survey, we wanted to avoid any bias. Many of the tests and questionnaires used in our center have been developed and/or validated by our own investigations.

Descriptive statistics were performed using IBM SPSS 22.

\section{Results}

Completed questionnaires were received from 25 reference centers corresponding to a response rate of $96 \%$.

\section{1. Neuropsychological service}

Characteristics of the participating centers are shown in Table 1. Epilepsy surgery is performed in-house in 23/25 centers (in two cases, epilepsy patients are referred for surgery to a collaborating clinic after presurgical work-up). In-house neuropsychological assessment is available in $23 / 25$ of the centers, two centers have external collaborating institutions. In those two centers without in-house neuropsychology, information derives from the collaborating institutions offering the neuropsychological service. All but one center regard the neuropsychological assessment as an important and indispensable part of the presurgical diagnostic work-up.

please insert here: Table 1. Characteristics of participating centers 
While in six centers the neuropsychological service is focused only on those with epilepsy, most clinics provide their service additionally for neurological, neurosurgical and other patient groups. Psychiatric service is provided in $92 \%$ of the centers, the evaluation is performed exclusively by psychiatrists $(60 \%)$, exclusively by psychologists $(4 \%)$, both by psychologists and/or psychiatrists (28\%), or both by psychiatrists and/or neurologists (4\%). Standardized inventories for the assessment of depression, anxiety and other psychiatric symptoms are used by $75 \%$ (valid answers $n=16$ ). The average time needed for a full presurgical evaluation is 4.5 hours, ranging between 1.5 and 10 hours.

\subsection{Neuropsychological assessment}

Table 2 shows data on the specifications of the neuropsychological assessments. Indications for neuropsychological assessment included localizing (100\%), in all cases presurgical $(100 \%)$ and all but one centers postsurgical (96\%) testing, the evaluation of general cognitive performance (92\%), monitoring antiepileptic drug (AED) therapy (68\%), testing for transient cognitive impairments (TCl) due to electroencephalographic epileptic discharges (56\%), as well as for ictal (20\%) and postictal (12\%) deficits.

Neuropsychological testing is used for clinical decision making i.e. for or against surgery $(88 \%)$, to determine the extent of surgery $(36 \%)$, to optimize antiepileptic drug treatment $(32 \%)$, to determine the need for cognitive rehabilitation $(32 \%)$ or for psychiatric treatment (24\%). These answers were given in response to an open question, so these data might not be exhaustive and may be under-representative of the actual frequencies.

The centers largely agree that memory, language, attention and executive functions need to be assessed as part of a surgical evaluation. There is also agreement about using normalized and standardized tests, employing either national or international normative data. Few clinics use their own unpublished norms. 
please insert here: Table 2. Neuropsychology: who, why, what - and what for?

Neuropsychological assessment for pediatric patients is provided in $76 \%$ of the centers (Table 1). Of 18 valid answers, the majority of centers use standardized developmental scales or tests already in babies and toddlers (Table 3). Of 23 centers offering in-house surgery, 19 (83\%) provide a surgical program for children. Of those, 17 provide neuropsychological assessment for children. Two centers provide neuropsychological assessment for children but do not offer in-house surgery at all (1) or not for children (1).

please insert here: Tab.3: Use of standardized tools for pediatric patients given in percentages

3.3. Which test instruments are currently used? Neuropsychological tests and questionnaires

With a total number of 168 different tests and procedures in use in children and adults, the variation in neuropsychological diagnostics between centers was considerable. Table 4 lists only those tests which are used by at least 3 centers. Multiple answers were possible for each domain. Tests are listed separately for children and adults. Concerning children, the results showed that development and IQ play an important role. Performance testing of is nevertheless also an issue in children and adolescents. Table 5 provides the source for justifying the selection of this specific test. As for references for the different tests see compendia like $[30,31]$ or see the websites of Pearson Assessment (http://www.pearsonassessment.de/), publisher Hogrefe (http://www.testzentrale.de/) and Psytest (http://www.psytest.net/index.php?page=TAP-2-2\&hl=en_US).

For testing of intelligence, among 7 different scales, the Wechsler Scales for children and adults was used in all except one center. For adults, either full (90\%) and/or abbreviated versions (15\%) are employed. In all three participating German-speaking centers, a 
vocabulary test is used for assessing crystallized and education dependent intelligence in adults (Mehrfachwahl-Wortschatz-Intelligenztest, MWT-B). For children, Wechsler scales for school (60\%) or preschool (33\%) age are applied.

For developmental diagnostics, the most popular scale is the Bayley Scales of Infant Development (33\%), while some centers use the Denver Developmental Screening test or, to measure adaptive behavior, the Vineland Adaptive Behaviour Scale (VABS) or the (20\% each).

For nonverbal memory functions, 10 different tests are in use. The Rey-Osterrieth Complex Figure test (ROCFT) is used most frequently (55\%) followed by only a few centers using the Diagnosticum für Cerebralschädigung (DCS-R) and Benton test. As for verbal memory functions, among 12 different tests, the Rey Auditory Verbal Learning Test is used frequently (RAVLT, $40 \%$ adults, $20 \%$ children), followed by the Logical Memory subtest of the Wechsler Memory Scale (WMS-R) for adults. Complete memory batteries are rarely used (WMS and Recognition Memory Test, RMT).

For children, no consensus of more than two centers was achieved for memory testing. Most frequently used are the DCS-R and as well the RAVLT (20\% each).

Short-term and working memory are tested with the Digit Span and Corsi Block-Tapping test by $70 \%$ of the centers for adults. These tests are also popular in children $(27 \%$ block tapping, 40\% digit span).

As for attentional functions, Trail Making Test (TMT) is applied by more than half of the centers for testing visual psychomotor speed and task switching. Other, less frequently used attentional tests in adults are the letter cancellation test D2 (Aufmerksamkeitsbelastungstest) in adults (30\%) and the Testbatterie zur Aufmerksamkeitsprüfung (TAP, 15\%). Executive functions are assessed by the Stroop paradigm (45\%) or Wisconsin Card Sorting Test (WCST, 15\%). Agreement for children however is lower, with only TMT reaching a consensus of $27 \%$. More popular (33\%) is the use of subtests from the Developmental Neuropsychological Assessment (NEPSY), which is also used for memory (2 centers), language ( 2 centers), motor ( 2 centers) and visuospatial functions ( 1 center). 
27 different language tests are in use. The majority of centers (75\% for adults, but only $33 \%$ for children) are using the Boston Naming Test, which is a word finding test. Although there was some critique uttered in our surveys (obsolete items, items dependent on education), there is no established alternative test so far. Additionally, many centers assess phonemic (45\%) and/or semantic (40\%) fluency for adults. As for receptive language functions, the subtest "token test" from the Aachener Aphasietest (AAT) is used widely ( $35 \%$ for adults, $33 \%$ for children).

Visuospatial function is assessed by 21 different tests, with little overlap. The most popular test is the copy trial of the ROCFT (35\%) which itself is also used as a figural memory test. All other tests are employed by $20 \%$ (Wechsler block design, line orientation) or $15 \%$ of centers (Hooper Visual Organization Test (VOT), Visual Object and Space Perception Battery, VOSP, Benton facial recognition). In children, little agreement (20\%) was found for the Developmental Test of Visual-Motor Integration (Beery VMI).

As for motor function, 18 different tests are in use. Agreement was low for adults and nonexistent for children. For adults, the most popular are the finger tapping (25\%), Luria Sequences (25\%) and the Purdue Pegboard (15\%). For children, no consensus in at least 3 centers was found.

The monitoring of emotions and behavior plays a crucial role in patients with epilepsy, given the high incidence of depression, anxiety, aggressive behavior and organic brain syndrome due to the epilepsy alone or its treatment. In adults, the Beck's Depression Inventory (BDI or BDI-II) is used in $60 \%$ of the clinics, followed by the Hospital Anxiety and Depression Scale (HADS) (25\%), Symptom Checklist 90-R (SCL 90-R, 20\%), Minnesota Multiphasic Personality Inventory (MMPI, 20\%) and Beck Anxiety Inventory (BAI (15\%)). For children, most frequently named was the Child Behavior Checklist (CBCL), used by $20 \%$ of the centers.

The ultimate goal of treating epilepsy is improving the individual quality of life. For adults, in $45 \%$ of the centers, the Quality Of Life in Epilepsy Inventory (QOLIE) is used in different versions (QOLIE-10, QOLIE-31, QOLIE-89). In three centers, the Short Form 36 (SF 36) is 
used, and three other inventories in single clinics. No agreement was found for pediatric assessment.

please insert here: Tab.4a: Tests used in the different centers for children and adolescents

The normative data of tests and questionnaires used in the centers are mostly a variation of published national $(92 \%)$, international $(79 \%)$ or local data $(33 \%)$. Some centers use unpublished local normative data (16\%). In general, only $52 \%$ of the centers $(n=13)$ provided us with sources for these normative data (for children and adults). Sources for validity (proof that the tests measures what it intends to measure) were provided by $40 \%(n=10)$ of the respondents. However, these sources were not always specific for use in patients with epilepsy. Only 5 centers (20\%) provided sources with proven evidence for use of the tools in patients with epilepsy. Another 5 stated that all tests are evidence-based but without citing any sources. We evaluated each of these sources given for validity in detail, considering only sources for those tests that are used by at least 3 centers. For adults, 38 sources were given for validity. These stem from the manual in $36.8 \%$ of given references, from epilepsyunspecific publications in $55.3 \%$ and from publications in epilepsy by $7.9 \%$. Except for one WAIS reference, the latter references were again in regard to the QOLIE inventory. In regard to children and adolescents, as for the validity of the tests in general or in epilepsy, 12 sources were given. $58.3 \%$ of these sources were test manuals, $8.3 \%$ publications apart from epilepsy and 33.3\% epilepsy-related publications.

In response to the question which areas of assessment would require further improvement, several aspects were indicated. These included more national norm data, a better test for the assessment of nonverbal memory in relation to right temporal lobe epilepsy, time efficient tests because of limited personal ressources, RAVLT and DCS co-normalization norms for comparison of lateralized temporal lobe functions and updated QOL questionnaires. Another question asked if there were any un-captured functions or behaviors seen in the patients for 
which a test would be desired. Suggestions were made for evaluating accelerated long-term forgetting, frontal lobe dysfunctions, divided visual field evaluation, every day performance and external validity of objective performance, mood and behavioral aspects (panic disorder, reaction to stress, organic brain syndrome, social cognition), and a qualitative scoring of test performance (type of mistakes, performance pattern). Although some tests would allow a more detailed analysis, corresponding scores are not yet part of a standard diagnostic routine and there is a need for further validation and standardization of these measures.

\subsection{Assessment of hemispheric dominance}

Assessing the dominant hemisphere for language functions is mostly performed by fMRI (in $91 \%)$ and IAT (59\%), combined with lateralizing tests (91\%) such as verbal vs. nonverbal memory and inventories (handedness) (Table 5). In single centers, Transcranial Magnetic Stimulation (TMS), Magnetoencephalograpy (MEG) and Diffusion Tensor Imaging (DTI) (arcuate tract) is performed for some cases. Functional transcranial Doppler sonography (fTCD) is used by two centers on a regular basis, with approximately $n=33$ and $n=51$ patients per year, one center using the "Bishop Picture Animation Paradigm" and one center using a word generation and picture description task.

please insert here: Table 5. Methods for assessing hemispheric dominance for language functions

The IAT, although long regarded as the gold standard for evaluation of hemispheric dominance, has increasingly been replaced by noninvasive methods [32]. The indications differ among the centers (Table 6), from performing the IAT in all cases of TLE of the dominant hemisphere to cases only with the epileptogenic focus in or adjacent to eloquent cortex $(n=5)$. As for the general approach, bilateral injection in the $\mathrm{ACl}$ is still the standard 
procedure. Anesthetics differ among the centers as well, with amobarbital used most frequently (69\%) but in some cases, propofol or methohexital.

Please insert here: Table 6. Protocols and methods for performing functional magnetic resonance imaging and the intracarotid amobarbital test for assessing the hemispheric dominance for language functions

Out of 20 participants with valid answers concerning fMRI, $20 \%$ always perform fMRI in presurgical patients, $55 \%$ perform $\mathrm{fMRI}$ if the resection is planned in or adjacent to an eloquent areal, $50 \%$ if atypical dominance is suspected, $30 \%$ if the to-be-resected lesion is located in the (presumably) dominant hemisphere, and 5\% perform $\mathrm{fMRI}$ in bitemporal cases (multiple answers were possible). Most centers use two (42\%) or three language paradigms $(37 \%)$, the minority use only one paradigm (21\%). Paradigms are mostly word generation (79\%), followed by naming (42\%), semantic comparisons (37\%), listening (32\%) and reading (26\%). Hemispheric dominance is assessed purely visually $(50 \%, n=7)$ or additionally with a lateralization index $(50 \%)$. Of the centers that use $\mathrm{fMRI}$ on a regular basis, SPM is most often used for analysis (54\%, 7 centers), other programs were used by one (FSL, BrainVoyager, BrainWave, Neuro3D) or two centers each (NordicBrainEx). Only two out of 25 centers provided the requested decision tree in this regard.

\section{Discussion}

Our survey collected information about the practice of neuropsychological assessments in 25 epilepsy centers and confirms that neuropsychological assessment is generally perceived as an essential part of the pre- and postsurgical work-up in children, adolescents and adults.

In most E-PILEPSY associated centers, neurosurgery and neuropsychology are performed in-house or close by, so that the conditions are optimal for short communication pathways and multi-disciplinary patient care. 
According to the survey, major indications for neuropsychological evaluations are the assessment of the general performance level, assessment of partial functions as well as ictal and postictal testing for localization diagnostics (relationships of structure and focus to function), determination of language dominance, negative cognitive antiepileptic drug effects, postsurgical follow-up and outcome control, and indication for rehabilitative care. In children, evaluation and monitoring of neurodevelopment is also a major indication. As for the structural and methodological aspects of neuropsychological assessment in epilepsy, the EU centers largely are in line with recent recommendations by the ILAE neuropsychology task force [20].

In times of limited personnel capacity (neuropsychological staff consists on average of 2.5 psychologists, with an average number of 171 patients tested per year and an average time of 4.5 hours for a presurgical evaluation, see Table 2.), it seems impossible to perform a thorough neuropsychological assessment with every patient receiving all tests and procedures. Instead a core test battery should be compiled which can be extended optionally depending on specific questions in terms of a modular diagnostics.

Looking at the domains that the centers rated as most essential there was a high consensus (64-90\%) regarding the necessity to assess memory, language, attention and executive functions. The order of the listing corresponds to the fact that most surgical candidates have temporal lobe epilepsy (memory \& language) followed by frontal and posterior epilepsies (attention, executive functions, language, visuo-construction, motor functions). Tests for primary and secondary posterior perceptual (optical, auditory, tactile functions) were largely not considered and are probably less relevant. IQ interestingly was considered as necessary by less than one third of the centers for children and adults $(5 / 18,27.8 \%)$, but for $75 \%(3 / 4)$ of centers testing only children. In young children developmental scales are widely used, and assess the major domains of psychomotor, cognitive and language development. Partial functions are relevant in children as well but as we know from the literature, localization and 
lateralization diagnostics are difficult when examining the not yet developed and specialized brain [1].

The overall number of all tests and questionnaires was vast $(n=168)$. However, following frequency statistics, good candidates for a core battery on which centers might agree could be found for most domains (see result section, 3.3). This selection is not meant to be a recommendation, but rather a reflection of the current practice. This is highly dependent on the availability of the individual tests in the specific countries or languages, and does not necessarily reflect the degree of evidence for the use of the tools in patients with epilepsy. For some countries, some consensus might already exist on a national level. In Germany, a working group with all neuropsychologists in epilepsy centers as well as a working group for presurgical diagnostics and epilepsy surgery associated with the national ILAE chapter has been established which meet at regular intervals and which are dedicated to harmonizing and improving assessments in Germany [27, 28, 33].

Neuropsychological assessment should not be an end in itself. It should provide information about the patients that is useful for clinical decision making. Neuropsychological testing was noted to be important in decision-making by influencing therapeutic decisions, counseling of patients in regard to possible cognitive sequels of surgery as well as determining the extent and timing of the surgery. Apart from the presurgical work-up per se, neuropsychology also helps to optimize treatment with AEDs, indicates the necessity to assess language dominance in cases of atypical memory profiles, leads to referrals to rehabilitation programs in case of significant postsurgical impairments, indicates the necessity of psychiatric care and treatment in cases of severe depression, psychosis, or mental decline/dementia, or indicates the initiation and/or termination of immune-modulatory therapies (in cases auf autoimmune encephalitis). Apart from exhaustive assessment, postictal testing of basic cognitive functions is one important aspect of presurgical diagnostic, indicating side or site of 
epileptogenic seizure focus [32]. This includes testing of language functions for lateralization of seizure origin.

Given these consequences of neuropsychological evaluations for patients and treatment decisions, it is well understood that the tests in use are valid and reliable in people with epilepsy. Purely performance-orientated testing does not necessarily meet the requirements of a sophisticated presurgical evaluation in epilepsy patients and might even be irresponsible if such non-specific knowledge is used for counseling, or when expected or predictable deficits cannot be detected or predicted. As a well-known example, with only IQ testing performed in the amnestic patient H.M. the catastrophic outcome of bilateral amygdalohippocampectomy would have been missed [34].

This was the reason all centers were asked to cite the sources which would demonstrate the validity of the used tests in regard to detecting the effects of epileptogenic lesions, seizures/epileptic activity, epilepsy surgery and pharmacological treatment. Here many sources were cited concerning the normalization and general validity of the tools used, i.e. that a memory test is indeed measuring memory and that it has age-based norms etc., but surprisingly few references were given addressing the validity of these tests in epilepsy. Either this means that the test selection is not based on evidence for the use in epilepsy, that there is no evidence, or that this question was skipped because lack of time, or simply because of a limited number of adequate tests available in certain centers.

Whatever the reason for the present results may be, the results parallel those of a national survey [24] which showed that only one third of the tests were evidence-based for use in epilepsy, another third by the fact that the tests are well normalized and standardized independent of epilepsy, and one third by personal preferences.

The second part of this study addressed hemispheric dominance assessment. It showed a high agreement on the methods in use. Almost all centers use fMRI in addition to lateralizing cognitive tests and handedness inventories. Many centers still use the IAT [35] but only in 
single selected cases and not as part of standard care. Generally there appears to be some uncertainty regarding the individual use and application of measures for the determination of dominance tests. This is at least indicated by the lack of agreement on the circumstances which would indicate the necessity of IAT and fMRI, reflecting what these methods are really good for. The rare use of the IAT reflects the steady decline of indications for the IAT which is reported in the literature. The remaining few cases probably show when this information is really indispensable and what this method is able to tell and predict. The future of $\mathrm{FMRI}$ and other methods and their value for presurgical diagnostics needs to be further explored.

\section{Conclusion}

The general principles of neuropsychological testing were evaluated across Europe and are consistent as such with what has recently been formulated by the neuropsychology task force of the ILAE [20]. This is encouraging since this knowledge provides a foundation that will inform future recommendations on which tests (minimum standards) should be used in the pre- and post-surgical care of persons with epilepsy. Similar to Marilyn Jones-Gotman's milestone work 23 years ago [21] however, there is still significant heterogeneity between centers in regards to the tests that are used. Nevertheless a subset of tests can be highlighted for the most essential cognitive and behavioral domains that appear to be good candidates for a core battery across the E-PILEPSY associated centers. Since only a minority of the tests in use had been or could be justified on the basis of published evidence, the essence of the survey shows that the final selection of tests will need to be based on the best evidence (ongoing systematic review), the current survey and for many, based on expert consensus methodology (until further evidence is available). Finally, as regards to the assessment of hemispheric dominance, lack of common paradigms raises the question as to when these results are really essential for clinical decision making. Here additional work is required to compare methods and paradigms to reveal the exact way of decision making in the individual patient. 


\section{Disclosure}

Dr. Helmstaedter reports personal fees from Glaxo Smith Kline, UCB Pharma, Pfizer, Desitin Pharma, EISAI, Viamed GmbH, Assurance companies, Courts, Occupational insurance associations, grants from German Research Foundation, all that are outside the submitted work. Dr. Philippe Ryvlin has received speaker or consultant fees from UCB pharma, Eisai, Cyberonics and Medtronics. Ms Vogt reports personal fees from the European Commission, Executive Agency for Health and Consumers, during the conduct of the study. All other authors have no conflicts of interest to disclose.

\section{Funding}

This report arises from the project E-PILEPSY that has received funding from the Executive Agency for Health and Consumers.

\section{Acknowledgement}

Acknowledged are the collaborators of the centers for contributing data and/or critically revising the manuscript: Ema Bran, Danguole Dadurkeviciene, Slavka Galic, Alice Maulisova, Lolia Pope, Martha Spilioti, Gergana Todorova. We thank Nathalie Jetté and Christian Hoppe for critically revising the manuscript.

\section{References}


[1]. Mouthaan BE, Rados M, Barsi $P$, et al. Current use of imaging and electromagnetic source localization procedures in epilepsy surgery centers across Europe. Epilepsia. 2016 57: 770-776.

[2]. Kobulashvili T, Hofler J, Dobesberger J, et al. Current practices in long-term videoEEG monitoring services: A survey among partners of the E-PILEPSY pilot network of reference for refractory epilepsy and epilepsy surgery. Seizure. 2016 38: 38-45.

[3]. Helmstaedter C, Witt JA. Clinical neuropsychology in epilepsy: theoretical and practical issues. Handbook of clinical neurology. 2012 107: 437-459.

[4]. Aikia M, Salmenpera T, Partanen K, Kalviainen R. Verbal Memory in Newly Diagnosed Patients and Patients with Chronic Left Temporal Lobe Epilepsy. Epilepsy Behav. 2001 2: 20-27.

[5]. Sherman EM, Wiebe S, Fay-McClymont TB, et al. Neuropsychological outcomes after epilepsy surgery: Systematic review and pooled estimates. Epilepsia. 2011 52: 857-869.

[6]. Helmstaedter C. Cognitive outcomes of different surgical approaches in temporal lobe epilepsy. Epileptic disorders : international epilepsy journal with videotape. 2013 15: 221239.

[7]. Bjellvi J, Flink R, Rydenhag B, Malmgren K. Complications of epilepsy surgery in Sweden 1996-2010: a prospective, population-based study. J Neurosurg. 2015 122: 519525.

[8]. Van Schooneveld MM, Braun KP. Cognitive outcome after epilepsy surgery in children. Brain Dev. 2013 35: 721-729.

[9]. Langfitt JT, Westerveld M, Hamberger MJ, et al. Worsening of quality of life after epilepsy surgery: effect of seizures and memory decline. Neurology. 2007 68: 1988-1994.

[10]. Helmstaedter C. Temporal lobe resection - does the prospect of seizure freedom outweigh the cognitive risks? Nature Clinical Practice Neurology. 2008 4: 66-67.

[11]. Boshuisen K, van Schooneveld MM, Uiterwaal CS, et al. IQ improves after antiepileptic drug withdrawal following pediatric epilepsy surgery. Annals of neurology. 2015.

[12]. Chelune GJ, Naugle RI, Luders H, Awad IA. Prediction of cognitive change as a function of preoperative ability status among temporal lobectomy patients seen at 6 -month follow-up. Neurology. 1991 41: 399-404.

[13]. Wilson SJ, Saling MM, Lawrence J, Bladin PF. Outcome of temporal lobectomy: expectations and the prediction of perceived success. Epilepsy Res. 1999 36: 1-14.

[14]. Helmstaedter CA. Prediction of memory reserve capacity. Adv Neurol. 1999 81: 271279.

[15]. Gross RE, Mahmoudi B, Riley JP. Less is more: novel less-invasive surgical techniques for mesial temporal lobe epilepsy that minimize cognitive impairment. Current Opinion in Neurology. 2015 28: 182-191.

[16]. Keezer MR, Sisodiya SM, Sander JW. Comorbidities of epilepsy: current concepts and future perspectives. The Lancet Neurology. 2016 15: 106-115.

[17]. Helmstaedter C, Aldenkamp AP, Baker GA, Mazarati A, Ryvlin P, Sankar R. Disentangling the relationship between epilepsy and its behavioral comorbidities - the need for prospective studies in new-onset epilepsies. Epilepsy \& behavior : E\&B. 2014 31: 43-47. [18]. Gilliam F, Kanner AM. Treatment of depressive disorders in epilepsy patients. Epilepsy \& behavior : E\&B. 2002 3: 2-9.

[19]. Cramer JA. Principles of health-related quality of life: assessment in clinical trials. Epilepsia. 2002 43: 1084-1095.

[20]. Wilson SJ, Baxendale S, Barr W, et al. Indications and expectations for neuropsychological assessment in routine epilepsy care: Report of the ILAE Neuropsychology Task Force, Diagnostic Methods Commission, 2013-2017. Epilepsia. 2015 56: 674-681.

[21]. Jones-Gotman M, Smith ML, Zatorre RJ. Neuropsychological testing for localizing and lateralizing the epileptogenic region. In: J E, ed. Surgical treatment of the epilepsies. New York: Raven Press, 1993: 245-262.

[22]. Rabin LA, Barr WB, Burton LA. Assessment practices of clinical neuropsychologists in the United States and Canada: a survey of INS, NAN, and APA Division 40 members.

Arch Clin Neuropsychol. 2005 20: 33-65. 
[23]. Djordjevic J, Jones-Gotman M. Inquiry on assessments across epilepsy centers in different countries. In: Helmstaedter C, Hermann B, Kahane P, Arzimanoglou A, eds. Neuropsychology in the Care of People with Epilepsy: John Libbey Eurotext, 2011. [24]. Witt JA, Helmstaedter C. A survey on neuropsychological practice in Germanspeaking epilepsy centers. In: Helmstaedter C, Hermann B, Kahane P, Arzimanoglou A, eds. Neuropsychology in the Care of People with Epilepsy: John Libbey Eurotext, 2011. [25]. Marilyn Jones-Gotman MLS, Robertt J. Zatorre. Neuropsychologigal Testing for Localizing and Lateralizing the epileptogenic region. In: Engel J, ed. Surgical Treatment of the Epilepsies: :. Lippincott Williams \& Wilkins, (1993). .

[26]. Helmstaedter C, Witt JA. Clinical neuropsychology in epilepsy: theoretical and practical issues. Handbook of clinical neurology / edited by PJ Vinken and GW Bruyn. 2012 107C: 437-459.

[27]. Witt JA, Helmstaedter C. Neuropsychology in Epilepsy Part II: Towards an Establishment of Diagnostic Guidelines. Fortschritte Der Neurologie Psychiatrie. 2009 77: 691-698.

[28]. Brückner K. Standard of neuropsychological testing

in presurgical epilepsy

diagnostics. Statement of

the neuropsychological

commission of the DGfE. Z Epileptol. 2012 25: 1-5.

[29]. Witt JA, Bodner T, Bruckner K, et al. Is There a Common Basis for Establishing Guidelines for the Neuropsychological Assessment in Epilepsy? A Survey across German Epilepsy Centres. Epilepsia. 2009 50: 45-45.

[30]. Lezak MD, Howieson DB, Bigler ED, Tranel D. Neuropsychological Assessement. 5th edn. Oxford: Oxford University Press, 2012.

[31]. Strauss E, Shermann EMS, Spreen O. A Compendium of Neuropsychological Tests: Administration, Norms, and Commentary

Oxford: Oxford University Press, 2006.

[32]. Baxendale S. The Wada test. Current Opinion in Neurology. 2009 22: 185-189.

[33]. Helmstaedter C, Witt JA. Neuropsychology in Epilepsy Part I: Cognitive Impairments in Focal Epilepsies. Fortschritte Der Neurologie Psychiatrie. 2009 77: 639-645.

[34]. Scoville WB, Milner B. Loss of recent memory after bilateral hippocampal lesions. 1957. The Journal of neuropsychiatry and clinical neurosciences. 2000 12: 103-113.

[35]. Haag A, Knake S, Hamer HM, et al. The Wada test in Austrian, Dutch, German, and Swiss epilepsy centers from 2000 to 2005: a review of 1421 procedures. Epilepsy Behav. 2008 13: 83-89. 